\title{
A Porous Crystal Plasticity Constitutive Model for Ductile Deformation and Failure in Porous Single Crystals
}

\author{
Amir Siddiq \\ School of Engineering, University of Aberdeen, UK \\ amir.siddiq@abdn.ac.uk
}

\begin{abstract}
This work presents a porous crystal plasticity model which incorporates the necessary mechanisms of deformation and failure in single crystalline porous materials. Such models can play a significant role in better understanding the behaviour of inherently porous materials which could be an artefact of manufacturing process viz. 3d metal printing. The presented model is an extension of the conventional crystal plasticity model. The proposed model includes the effect of mechanics based quantities, such as stress triaxiality, initial porosity, crystal orientation, void growth and coalescence, on the deformation and failure of a single crystalline material. A detailed parametric assessment of the model has been presented to assess the model behaviour for different material parameters. The model is validated using uniaxial data taken from literature. Lastly, model predictions have been presented to demonstrate the model's ability in predicting deformation, and failure in polycrystalline sheet materials.
\end{abstract}

Keywords Porous crystal plasticity, Void growth and coalescence, Metal Forming, Porous single metal crystals

\section{Introduction}

Depending upon the manufacturing process a metal can have inherent microvoids which can play a significant role during material deformation and failure. In the last two decades, researchers and scientists have worked on developing phenomenological theories that can be used to predict failure in metals during different loading conditions (for details please see ref [1]-[3] and therein). Based on the experimental findings [4], [5], a range of constitutive models have been developed in the past to account for void growth and coalescence to predict ductile failure in metals [1], [6], [7]. Siddiq et al. [1] presented a variational void coalescence model that includes all the essential ingredients of deformation and failure in ductile metals, i.e. elastic deformation, plastic deformation including deviatoric and volumetric (void growth) plastic deformation followed by damage initiation and evolution due to void coalescence. The model in general is an extension of variational J2-plasticity theory. McClintock [8] and Rice and Tracey [9] proposed void growth model for cylindrical and spherical voids, respectively. Gurson [10] proposed a model for spherical void with porosity the only microstructural variable. Koplik and Needleman [11], Tvergaard [12], Pardoen et al. [13] modified Gurson's model to define the damage initiation criterion based on maximum effective stress or the critical porosity. As discussed above, there is a whole family of constitutive models available that account for void growth and coalescence. All such models are based on the assumption that voids are present in the isotropic matrix and do not take into account crystalline anisotropy inside the individual grain due to different grain orientations. 
Recent crystal plasticity based representative volume element (RVE) simulations of single crystalline material with microvoids have shown the significant role played by crystal orientation during void growth under different types of loading [14]-[19]. Also, recent discrete dislocation simulations [20] and atomistic simulations [21] have shown that it is necessary to account for the effect of crystal anisotropy on void growth and coalescence. The above works suggests it is necessary to develop a crystal plasticity based constitutive model that accounts for the effect of mechanics based quantities, such as stress triaxiality, initial porosity, and crystal orientation inside individual grains, on the overall deformation and failure.

There has been significant research performed in this area in the recent past, these works have been thoroughly reviewed and discussed in the literature, for example by incorporating crystallographic aspect for anisotropic behaviour ([14], [22]-[26] and references there in) or anisotropy through phenomenological plasticity models (for details see [26]-[28]). These works are based on Gurson type approach (for details see [25]-[28] and references therein), and homogenisation approaches ([14], [22]-[24]).

In the proposed work, an approach similar to [1] has been used to incorporate the void growth and coalescence effect in a crystal plasticity based constitutive model in a phenomenological context. The model is capable of predicting the microstructure evolution during deformation and is also able to predict the onset and evolution of failure due to void growth and coalescence inside individual grains, i.e. intragranular ductile failure. The model accounts for the effects of crystal orientation, porosity, void growth and coalescence.

The paper is organised into 5 sections. Section 2 presents the void growth and coalescence model in crystal plasticity framework. Section 3 presents a detailed parametric assessment of the proposed model. Section 4 present the model validation and finite element based application. Final conclusions and future directions are presented in section 5.

\section{Crystal Plasticity Framework}

\subsection{Kinematics}

Conventional crystal plasticity theory has been extended to account for the elastic-plastic response due to crystallographic slip and void growth. The total deformation gradient is described through multiplicative decomposition of the deformation gradient $(\boldsymbol{F})$ into elastic $\left(\boldsymbol{F}^{\boldsymbol{e}}\right)$ and a plastic $\left(\boldsymbol{F}^{\boldsymbol{p}}\right)$ component.

$$
F=F^{e} F^{p}
$$

Based on Figure 1, the elastic part $\left(\boldsymbol{F}^{\boldsymbol{e}}\right)$ can be decomposed into the symmetric left elastic stretch tension $\left(\boldsymbol{U}^{\boldsymbol{e}}\right)$ and the rotation tension $\left(\boldsymbol{R}^{\boldsymbol{e}}\right)$.

$F^{e}=U^{e} R^{e}$

Thus kinematics of the single crystal is given by

$F=U^{e} R^{e} F^{p}$

Here $\boldsymbol{R}^{\boldsymbol{e}} \boldsymbol{F}^{\boldsymbol{p}}$ is the denoted by $\boldsymbol{F}^{*}$ representing the plasticity and rotation of the lattice and based on Figure 1 is given by 
$F^{*}=R^{e} F^{p}=R^{e} F_{s}^{p} F_{v}^{p}$

where $\boldsymbol{F}_{\boldsymbol{s}}^{\boldsymbol{p}}$ is the plastic deformation gradient due to plastic slip while $\boldsymbol{F}_{\boldsymbol{v}}^{\boldsymbol{p}}$ is the plastic deformation gradient due to void growth.

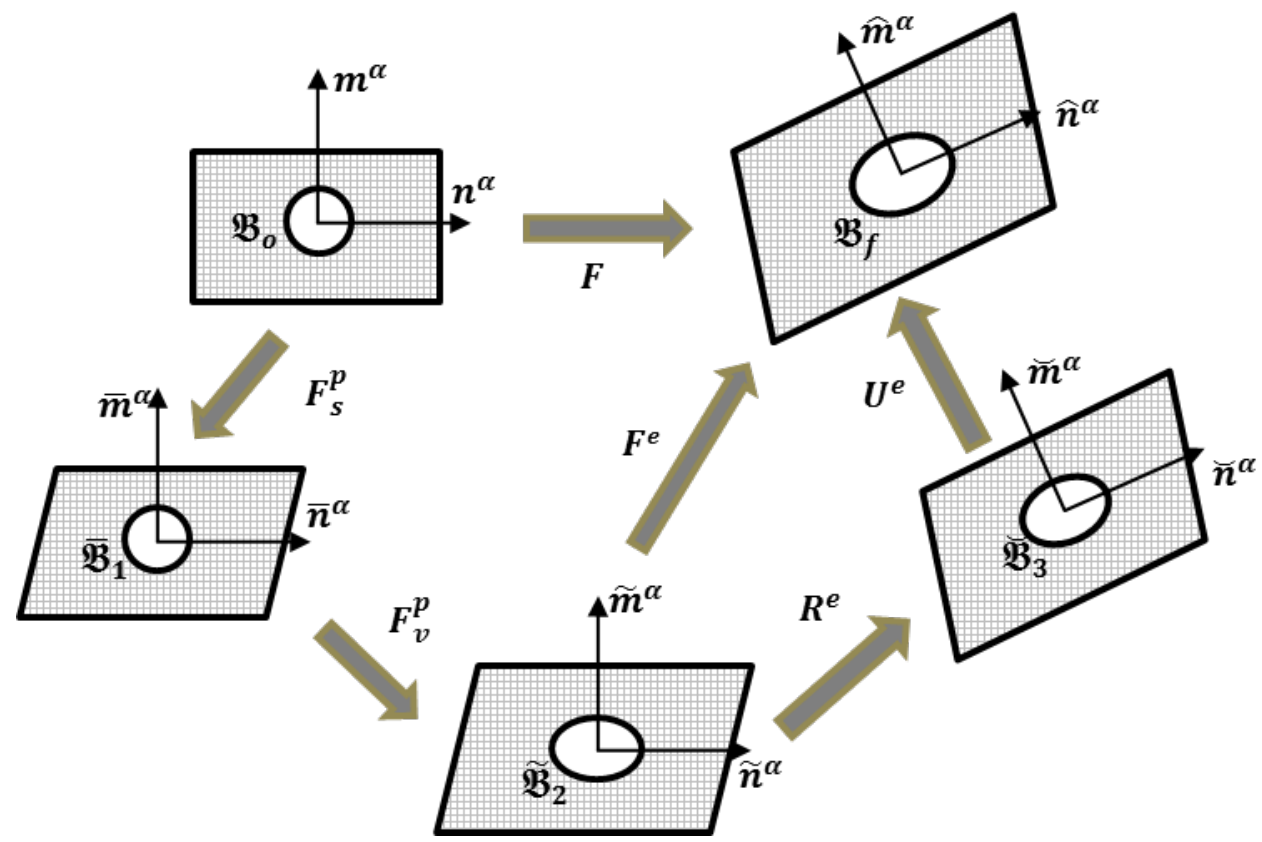

Figure 1: Elastic-plastic deformation kinematics of single crystals with voids by crystallographic slip and void growth

The velocity gradient $(\boldsymbol{L})$ in current configuration $\mathfrak{B}_{f}$ is given by

$L=\dot{\boldsymbol{F}} \boldsymbol{F}^{-1}=\dot{U}^{e}\left(\boldsymbol{U}^{e}\right)^{-1}+U^{e} \breve{L}^{*}\left(U^{e}\right)^{-1}$

Where $\widetilde{\boldsymbol{L}^{*}}$ is velocity gradient in $\breve{\mathfrak{B}}_{3}$ configuration and is given by

$\breve{L}^{*}=\dot{F}^{*} F^{*-1}=\dot{R}^{e} R^{e^{T}}+R^{e} \tilde{L}^{p} R^{e^{T}}$

with $\widetilde{\boldsymbol{L}}^{\boldsymbol{p}}=\overline{\boldsymbol{L}}_{\boldsymbol{s}}^{\boldsymbol{p}}+\widetilde{\boldsymbol{L}}_{\boldsymbol{v}}^{\boldsymbol{p}}$ being the plastic velocity gradient in $\widetilde{\mathfrak{B}}_{2}$ configuration and $\dot{\boldsymbol{R}}^{\boldsymbol{e}} \boldsymbol{R}^{\boldsymbol{e}}$ is the spin of the crystal.

The plastic velocity gradient $\left(\overline{\boldsymbol{L}}_{\boldsymbol{s}}^{\boldsymbol{p}}\right)$ in $\overline{\mathfrak{B}}_{1}$ is related to the slip rate $\left(\dot{\gamma}^{\alpha}\right)$ of the individual slip system by

$\overline{\boldsymbol{L}}_{\boldsymbol{s}}^{\boldsymbol{p}}=\dot{\boldsymbol{F}}_{\boldsymbol{s}}^{\boldsymbol{p}} \boldsymbol{F}_{\boldsymbol{s}}^{\boldsymbol{p}^{-\mathbf{1}}}=\sum_{\propto=1}^{N} \dot{\gamma}^{\alpha}\left(\overline{\boldsymbol{m}}^{\alpha} \otimes \overline{\boldsymbol{n}}^{\alpha}\right)$

with $\overline{\boldsymbol{m}}^{\alpha}$ and $\overline{\boldsymbol{n}}^{\alpha}$ define the orientation of the slip system $\alpha$ in $\overline{\mathfrak{B}}_{1}$ through Schmidt tensor $\left(\overline{\boldsymbol{S}}^{\alpha}=\overline{\boldsymbol{m}}^{\alpha} \otimes \overline{\boldsymbol{n}}^{\alpha}\right)$.

For plastic velocity gradient in $\widetilde{\mathfrak{B}}_{2}$ due to the void growth only, we use the following relationship

$\tilde{\boldsymbol{L}}_{\boldsymbol{v}}^{\boldsymbol{p}}=A \dot{\beta}_{g}\left(\frac{1}{3}\right) \boldsymbol{I}$ 
with $\dot{\beta}_{g} \geq 0$ for expansion while $\dot{\beta}_{g} \leq 0$ for contraction and $A$ is a material constant which scales the $\beta_{g}$. It must be noted that plastic velocity gradient due to void growth is assumed to be purely hydrostatic which is based on the assumption that void growth in FCC crystals is very small for deviatoric part of the deformation (see for example [29]) and previous constitutive models ([1], [7]). It must be noted that the effect of void growth on plastic slip (deviatoric part of plastic strain) is not demonstrated here. However, it will be shown later in section 2.3.2 that model does incorporate the effect of the presence of void and its growth on the plastic slip activity due to a combine effect of local stress concentrations, triaxialities and shape change as reported in literature (see for example [19], [24], [29]).

Substituting equations ( 7 ) and ( 8 ) into ( 6 ) and mapping the Schmidt tensors $\overline{\boldsymbol{S}}^{\alpha}$ in $\overline{\mathfrak{B}}_{1}$ and $\tilde{\boldsymbol{S}}^{\alpha}$ in $\overline{\mathfrak{B}}_{2}$ to $\breve{\mathfrak{B}}_{3}$ through forward transformation as $\breve{\boldsymbol{S}}^{\alpha}=\boldsymbol{R}^{e} \tilde{\boldsymbol{S}}^{\alpha}\left(\boldsymbol{R}^{e}\right)^{\boldsymbol{T}}=\boldsymbol{R}^{e} \overline{\boldsymbol{S}}^{\alpha}(\boldsymbol{R})^{\boldsymbol{T}}$ will yield

$\breve{\boldsymbol{L}}^{*}=\dot{\boldsymbol{R}}^{\boldsymbol{e}} \boldsymbol{R}^{\boldsymbol{e} \boldsymbol{T}}+\sum_{\propto=1}^{N} \dot{\gamma}^{\alpha} \breve{\boldsymbol{S}}^{\boldsymbol{\alpha}}+A \dot{\beta}_{g}\left(\frac{1}{3}\right) \boldsymbol{I}$

Hence $\breve{\boldsymbol{L}}^{*}$ describes the plastic flow due to crystallographic slip, the rotation of the lattice and void growth as referred to the unloaded configuration $\breve{B}_{3}$.

The velocity gradient $(\boldsymbol{L})$ in the current state $\left(\mathfrak{B}_{f}\right)$ can be decomposed as $\boldsymbol{L}=\dot{\boldsymbol{F}} \boldsymbol{F}^{-\mathbf{1}}=\boldsymbol{D}+\boldsymbol{W}$ where $\boldsymbol{D}=\operatorname{sym}(\boldsymbol{L})$ and $\boldsymbol{W}=\operatorname{skew}(\boldsymbol{L})$ are deformation and spin rate tensors, respectively. We can write the symmetric and skew parts of the velocity gradient $(\boldsymbol{L})$ as

$D=\operatorname{sym}\left(\dot{U}^{e} U^{e^{-1}}\right)+U^{e^{-T}} \breve{D}^{*} U^{e^{-1}}$

$W=\operatorname{skew}\left(\dot{U}^{e} U^{e^{-1}}\right)+U^{e^{-T}} \bar{W}^{*} U^{e^{-1}}$

with $\breve{\boldsymbol{D}}^{*}$ and $\widetilde{\boldsymbol{W}}^{*}$ in $\breve{\mathfrak{B}}_{3}$ configuration are defined as $\breve{\boldsymbol{D}}^{*}=\operatorname{sym}\left(\breve{\boldsymbol{C}}^{\boldsymbol{e}} \breve{\boldsymbol{L}}^{*}\right)$ and $\widetilde{\boldsymbol{W}}^{*}=\operatorname{skew}\left(\breve{\boldsymbol{C}}^{\boldsymbol{e}} \breve{\boldsymbol{L}}^{*}\right)$.

Using equation (6), $\breve{\boldsymbol{D}}^{*}$ and $\widetilde{\boldsymbol{W}}^{*}$ can be given by

$\breve{D}^{*}=\operatorname{sym}\left(\breve{C}^{e} \breve{\Omega}^{e}\right)+R^{e} \widetilde{D}^{p} R^{e T}$

$\widetilde{W}^{*}=\operatorname{skew}\left(\breve{C}^{e} \breve{\Omega}^{e}\right)+R^{e} \widetilde{W}^{p} R^{e T}$

with $\breve{\Omega}^{e}=\dot{\boldsymbol{R}}^{\boldsymbol{e}} \boldsymbol{R}^{\boldsymbol{e}^{T}}$ is the spin of the lattice and $\breve{\boldsymbol{C}}^{\boldsymbol{e}}=\boldsymbol{U}^{\boldsymbol{e}^{T}} \boldsymbol{U}^{\boldsymbol{e}}$ is right Cauchy-Green elastic strain tensor in configuration $\breve{\mathfrak{B}}_{3}$.

\subsection{Constitutive Model}

In the present work, a similar approach as proposed by [30] and later used by [31] has been used, i.e.

the relationship between the deformation rate $\left(\boldsymbol{D}^{\boldsymbol{e}}\right)$ and the Jaumann rate of Cauchy stress $(\tilde{\boldsymbol{\sigma}} \boldsymbol{\boldsymbol { \sigma }})$, which is given by

$\stackrel{\nabla^{e}}{\tilde{\sigma}}+\sigma\left(I: D^{e}\right)=\overline{\bar{C}}: D^{e}$ 
where $\overline{\overline{\boldsymbol{C}}}$ is the symmetric elastic tensor with $C_{i j k l}=C_{j i k l}=C_{i j l k}=C_{k l i j}$. It must be noted $\nabla^{e}$

that $(\tilde{\boldsymbol{\sigma}})$ rotate with the crystal lattice, which is related to corotational stress rate on axes rotating with the material (for details see [31]).

Using the same analogy as [30], [31], the resolved shear stress in slip system $\alpha$ can be related to the conjugate strain measure (plastic slip) for finite deformation as

$\tau^{\alpha}=\boldsymbol{\sigma}: \operatorname{sym}\left(\breve{\boldsymbol{S}}^{\alpha}\right)$

Substitution and simplification of equation ( 14 ) into ( 15 ) would give the relationship for the rate of change of resolved shear stress in slip system $\alpha$.

$\dot{\tau}^{\alpha}=\left(\widetilde{\boldsymbol{\sigma}}^{\boldsymbol{e}}+\boldsymbol{\sigma}\left(\boldsymbol{I}: \boldsymbol{D}^{\boldsymbol{e}}\right)-\boldsymbol{D}^{\boldsymbol{e}} \boldsymbol{\sigma}+\boldsymbol{\sigma} \boldsymbol{D}^{\boldsymbol{e}}\right): \operatorname{sym}\left(\breve{\boldsymbol{S}}^{\alpha}\right)$

\subsection{Specific Constitutive Relations}

Based on the discussion in the previous section, one now requires to prescribe the evolution equations for the slip $\left(\gamma^{\alpha}\right)$ in slip system $\alpha$, Evolution of the strength $\chi_{s}^{\alpha}$ of the slip system, hardening laws for lattice strain $\left(h_{\alpha \beta}\right)$, and void growth $\left(\beta_{g}\right)$.

\subsubsection{Evolution of Plastic Slip}

Plastic slip rate in slip system $\alpha$ in a rate dependent material can be estimated using the wellknown power law

$\dot{\gamma}^{\alpha}=\dot{\gamma}_{0}^{\alpha}\left[\frac{\left|\tau^{\alpha}\right|}{\chi_{s}^{\alpha}}\right]^{m_{1}} \operatorname{sgn}\left(\tau^{\alpha}\right)$

with $\dot{\gamma}_{0}^{\alpha}$ is the reference strain rate and $\chi_{s}^{\alpha}$ is the flow strength (strength of the slip system).

\subsubsection{Strength of Slip System Evolution}

Evolution of the strength of the slip system $\alpha$ is given in terms of plastic slip rate

$\dot{\chi}_{s}^{\alpha}=\sum_{\beta} h_{\alpha \beta}\left|\dot{\gamma}^{\beta}\right|$

with $h_{\alpha \beta}$ denotes the hardening moduli, and sum ranges over all activated slip systems.

A range of hardening functions (moduli) are available in the literature [3], [31], [32]. For the present work, a modified form a simple hardening moduli [32], [33] is used by including the early activation of single crystalline slip systems due to stress concentrations and void growth.

$h_{\alpha \alpha}=\varphi h_{0} \operatorname{sech}^{2}\left|\frac{h_{0} \gamma}{\tau_{s}-\tau_{0}}\right|$

$h_{\alpha \beta}=q h_{\alpha \alpha}$

Where $h_{\alpha \alpha}$ is self-hardening moduli, $h_{0}$ the initial hardening, $\tau_{0}$ the initial value of current strength, $\tau_{s}$ is the strength after which large plastic flow starts, $\gamma$ is the Taylor cumulative shear strain on all slip systems $\left(\gamma=\sum_{\alpha} \int_{0}^{t}\left|\dot{\gamma}^{\alpha}\right| d t\right.$ ), and $q$ is latent hardening constant. $\varphi$ is 
activation of slip systems due to void growth and stress concentration. For the present study, it is assumed to be $\varphi=e^{-s \beta_{g}}$ with $s$ being the material parameter characterising the slip system strength reduction or in other words stress concentration effect.

\subsubsection{Void Growth Rate}

For void fraction evolution we use a relationship motivated from representative volume element (RVE) based simulations [2] where evolution of a strain like internal variable $\left(\beta_{g}\right)$ due to void growth is given by

$\dot{\beta}_{g}=\frac{B}{\varepsilon_{h 0} m_{2}} \cdot\left[1+m_{2}\left(1-\frac{\varepsilon_{h}}{\varepsilon_{e q}}\right)\right]\left[\frac{\varepsilon_{h}}{\varepsilon_{e q}}\right]^{m_{2}}$

where $B, \varepsilon_{h 0}$ and $m_{2}$ are the material parameters. $B$ can be considered as the initial reference volumetric plastic strain due to void growth and for spherical voids can be given by

$B=f_{0} \dot{\beta_{0}}=\frac{4}{3} \pi r_{0}^{3} \cdot N_{0} \cdot \dot{\beta_{0}}$

with $f_{0}$ is the initial porosity and is given by $f_{0}=\frac{4}{3} \pi r_{0}^{3}$ for spherical voids, while $N_{0}$ is the initial void density and $\dot{\beta_{0}}$ is a parameter which represents the rate of void growth with reference to uniaxial loading and is assumed to be $\dot{\beta_{0}}=C \frac{\varepsilon_{e q}}{\varepsilon_{h}}$. Also, $\varepsilon_{h}=\log (\operatorname{det} \boldsymbol{F})$ is the hydrostatic strain, and $\varepsilon_{e q}$ is the equivalent strain. Both $\varepsilon_{h}$ and $\varepsilon_{e q}$ account for total deformation, i.e. elastic and plastic strains. $\varepsilon_{h 0}$ is the reference hydrostatic strain. For zero stresses or strains $\beta_{g}$ is set to be zero.

\subsubsection{Void Coalescence}

There has been models developed in the past which can simulate void coalescence using micromechanics based yield criterion which account for void coalescence by internal necking or internal shearing (see references [34]-[37] and there in). It has been reported in the literature [38] that onset of coalescence strongly depends on the initial orientation of the crystal with respect to loading axis. The orientation effect has been included in the void growth part and will be shown later that initial orientation governs how fast or slow $\beta_{g}$ grows. In this work a void coalescence model similar to [1] is used, it is assumed that as soon as $\beta_{g}$ reached to a critical value $\left(\beta_{g c}\right)$ the void growth will be taken over by void coalescence, i.e. $\beta_{g}$ is replaced by $\beta_{\text {coal }}$ throughout. In the mathematical terms, the strain like internal variable due to void coalescence $\left(\beta_{\text {coal }}\right)$ is given by

$\beta_{\text {coal }}=\beta_{g c}+\alpha_{1}\left(\beta_{g}-\beta_{g c}\right)^{\alpha_{2}}$

where $\alpha_{1}, \alpha_{2}$, and $\beta_{g c}$ are material parameters to be identified from experiments.

\subsection{Numerical Implementation}

This section presents an incremental solution procedure for the time integration of the constitutive equations of the porous crystal plasticity. Similar to [31], forward gradient time integration scheme is implemented in this work.

Plastic slip increment $\Delta \gamma^{\alpha}$ within the time increment $\Delta t$ can be defined as 
$\Delta \gamma^{\alpha}=\gamma^{\alpha}(t+\Delta t)-\gamma^{\alpha}(t)$

Using linear interpolation within $\Delta t$ would yield

$\Delta \gamma^{\alpha}=\Delta t\left[(1-\theta) \dot{\gamma}_{t}^{\alpha}+\theta \dot{\gamma}_{t+\Delta t}^{\alpha}\right]$

Subscript of $\dot{\gamma}_{t}^{\alpha}$ is the time at which it is computed. $\theta$ is integration parameter and ranges from 0 to 1 . For details please see ref [31].

As slip rate is a function of both resolved shear stress and strength of the slip system using Taylor expansion would lead

$\Delta \gamma^{\alpha}=\left[\dot{\gamma}_{t}^{\alpha}+\theta \frac{\partial \dot{\gamma}}{\partial \tau^{\alpha}} \Delta \tau^{\alpha}+\theta \frac{\partial \dot{\gamma}}{\partial \chi_{s}^{\alpha}} \Delta \chi_{s}^{\alpha}\right] \Delta t$

Increments of current strength of slip system $\left(\Delta \chi_{s}^{\alpha}\right)$, resolve shear stress $\left(\Delta \tau^{\alpha}\right)$ and void fraction due to growth $\left(\Delta \beta_{g}\right)$ are given by

$\Delta \chi_{s}^{\alpha}=\sum_{\beta} h_{\alpha \beta} \Delta \gamma^{\alpha}$

$\Delta \tau^{\alpha}=\left[C_{i j k l} \mu_{k l}^{\alpha}+\omega_{i k}^{\alpha} \sigma_{j k}+\omega_{j k}^{\alpha} \sigma_{i k}\right]\left[\Delta \varepsilon_{i j}-\sum_{\beta} \mu_{i j}^{\beta} \Delta \gamma^{\beta}\right]$

$\Delta \beta_{g}=\beta_{g, t+\Delta t}-\beta_{g, t}$

The co-rotational stress increment $\left(\Delta \sigma_{i j}\right)$ is finally given by

$\Delta \sigma_{i j}=C_{i j k l} \Delta \varepsilon_{k l}-\sigma_{i j} \Delta \varepsilon_{k k}-\sum_{\alpha}\left[C_{i j k l} \mu_{k l}^{\alpha}+\omega_{i k}^{\alpha} \sigma_{j k}+\omega_{j k}^{\alpha} \sigma_{i k}\right] \Delta \gamma^{\alpha}-C_{i j k l}\left[A \Delta \beta_{g}\left(\frac{1}{3} \delta_{k l}\right)\right]$

where $\mu_{i j}^{\beta}=\operatorname{sym}\left(\breve{\boldsymbol{S}}^{\alpha}\right)$ and $\omega_{i j}^{\alpha}=\operatorname{skew}\left(\breve{\boldsymbol{S}}^{\alpha}\right)$.

For a given strain increment $\left(\Delta \varepsilon_{k l}\right)$, the increments of void growth $\left(\Delta \beta_{g}\right)$ is computed using ( 28 ) while the increments of shear strain $\left(\Delta \gamma^{\alpha}\right)$ in the slip systems are uniquely calculated by substituting equation ( 26 ), ( 27 ) and ( 28 ) into ( 25 ). Once $\Delta \gamma^{\alpha}$ and ( $\Delta \beta_{g}$ ) are known in terms of the strain increments $\left(\Delta \varepsilon_{i j}\right)$, all the other increments can be found through equations ( 26 ), ( 27 ) and ( 29 ).

\section{Parametric Assessment of the Model}

Parametric assessment of the model is being presented in this section. Aim of this assessment is to present the effect of individual model parameters on the stress-strain response for different types of loading. As discussed in the previous section, total deformation of single crystal comprises of the elastic part due to lattice stretching, plastic deformation due to plastic slip in individual slip system and void growth contributions, and lastly the damage initiation and evolution due to void coalescence. Parametric studies have been carried for uniaxial and volumetric (triaxiality $=1,3$ ) loading to understand the effect of void growth and coalescence parameters on stress -strain response. 
Table 1: Material parameters associated with void growth and coalescence used for parametric assessment

\begin{tabular}{lccccccc}
\hline $\mathrm{B}$ & $\varepsilon_{h 0}$ & $\mathrm{~m}_{2}$ & $\mathrm{~s}$ & $\mathrm{~A}$ & $\beta_{g c}$ & $\alpha_{1}$ & $\alpha_{2}$ \\
0.0001 & 3.0 & 5.0 & 1000 & 1.0 & 0.0001 & 1.0 & 1.0 \\
\hline
\end{tabular}

Numerical tests have been performed systematically by varying (one by one) firstly the five material parameters $\left(A, B, \varepsilon_{h 0}, m_{2}, s\right)$ associated with void growth. Later on the three material parameters $\left(\alpha_{1}, \alpha_{2}, \beta_{g c}\right)$ which account for void coalescence are assessed. The rest of the material parameters are kept constant and are given in Table 1.

Figure 2 shows the effect of different material parameters $\left(A, B, \varepsilon_{h 0}, m_{2}, s\right)$ associated with void growth for uniaxial test case, where volumetric stress is $\operatorname{tr}(\boldsymbol{\sigma})$ and volumetric strain is $\log (\operatorname{det} \boldsymbol{F})$. Figure 2(a) shows the effect of $A$ on the stress-strain response. $A$ is first introduced in equation (8) for transforming and scaling the internal variable $\left(\beta_{g}\right)$ associated with void growth into a strain like variable. Presented model has been assessed for a range of $A$ values from 0.1-10. It can be inferred from Figure 2(a) that the increasing value of $A$ reduces the slope of the softening part of the stress-strain response. It is also found that the softening initiates earlier as the value of $A$ is increased. Effect of variation of parameter $B$ on stress-strain response is shown in Figure 2 (b). $B$ is the initial reference volumetric plastic strain and was introduced in equation (23). It is found that increasing value of $B$ also reduces the slope of the softening part of the stressstrain response along with the reduction of damage initiation strain and strength. Overall, for both $A$ and $B$ resulted into the softer stress-strain response and earlier softening due to void growth with increasing values.

$\varepsilon_{h 0}$ and $m_{2}$ were material parameters first introduced in equation (23) with $\varepsilon_{h 0}$ being the reference hydrostatic strain. Effect of $\varepsilon_{h 0}$ and $m_{2}$ is presented in Figure 2 (c) and (d), respectively. It is found that as the value of $\varepsilon_{h 0}$ is increased the softening effect diminishes and damage initiation (void growth) strength and strain increases. For $m_{2}$ as the value is increased the slope of the softening part of the stress-strain curves increase without significantly effecting the strength at which void growth becomes significant causing faster softening. $s$ being the material parameter characterising the slip system strength reduction or in other words stress concentration effect was first introduced in the context of equation (22). It is found from Figure 2 (e) that as the value of $s$ is increased the stress-strain response seems to be unaffected, however $\beta_{g}$ evolved faster and its critical value $\left(\beta_{g c}\right)$ was reached earlier with increasing value of parameter $s$. 

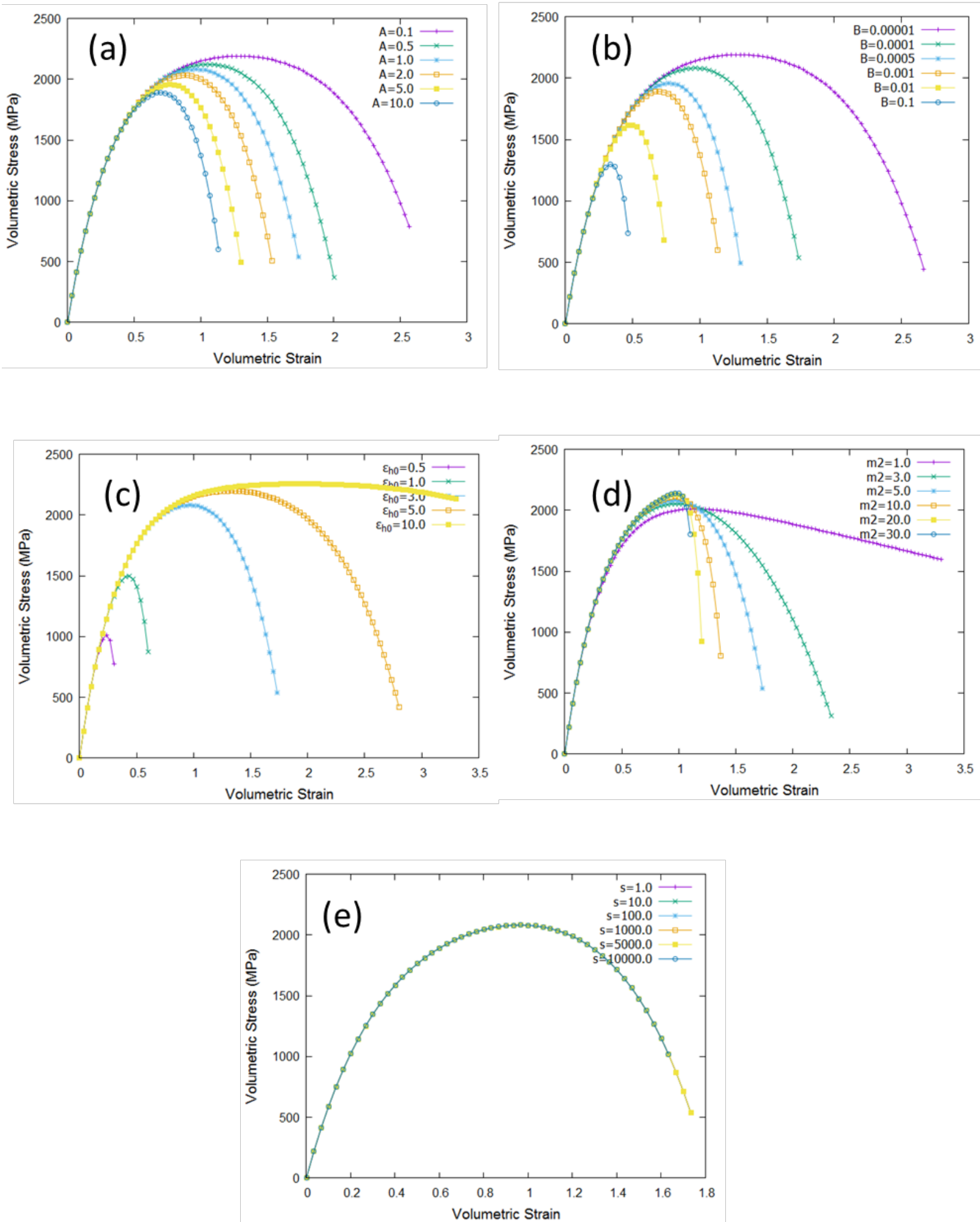

Figure 2: Parametric study for the case of uniaxial loading with void growth only: Effects of (a) $A$, (b) $B$, (c) $\varepsilon_{h 0}$, (d) $\boldsymbol{m}_{2}$, (e) $s$ 

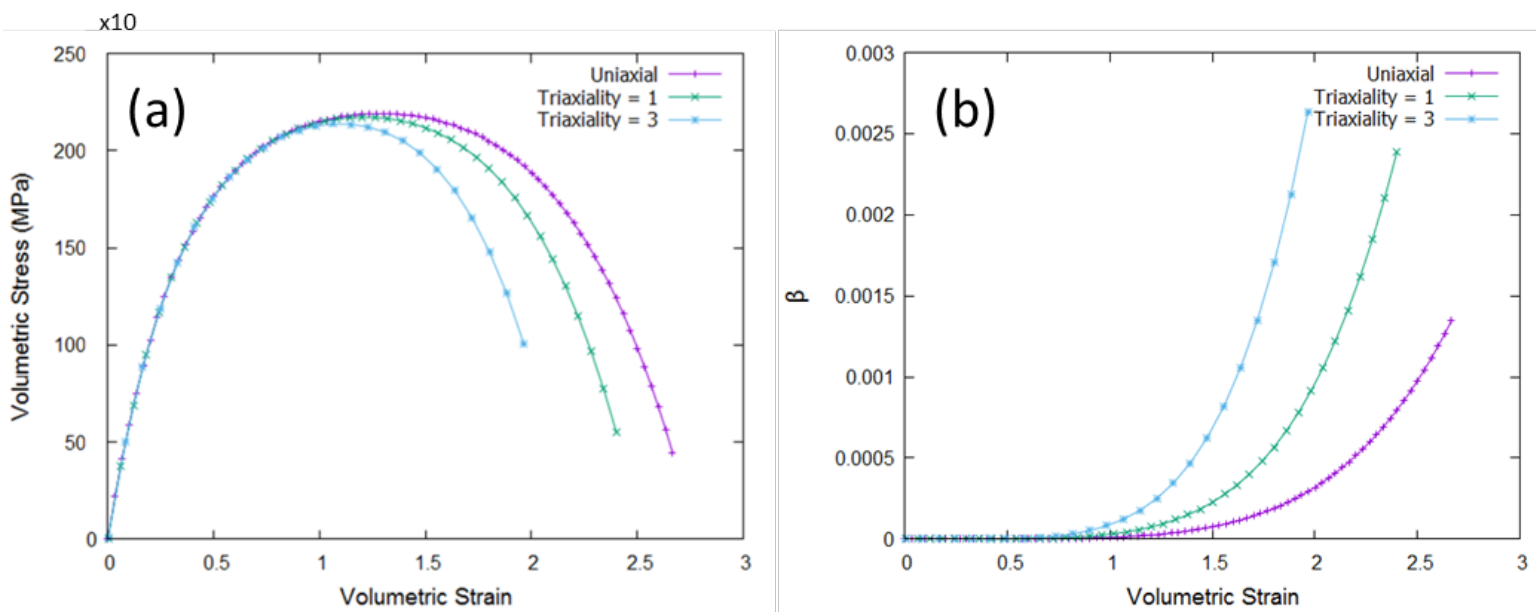

Figure 3: Effect of Triaxiality on the stress-strain response and plastic strain due to void growth
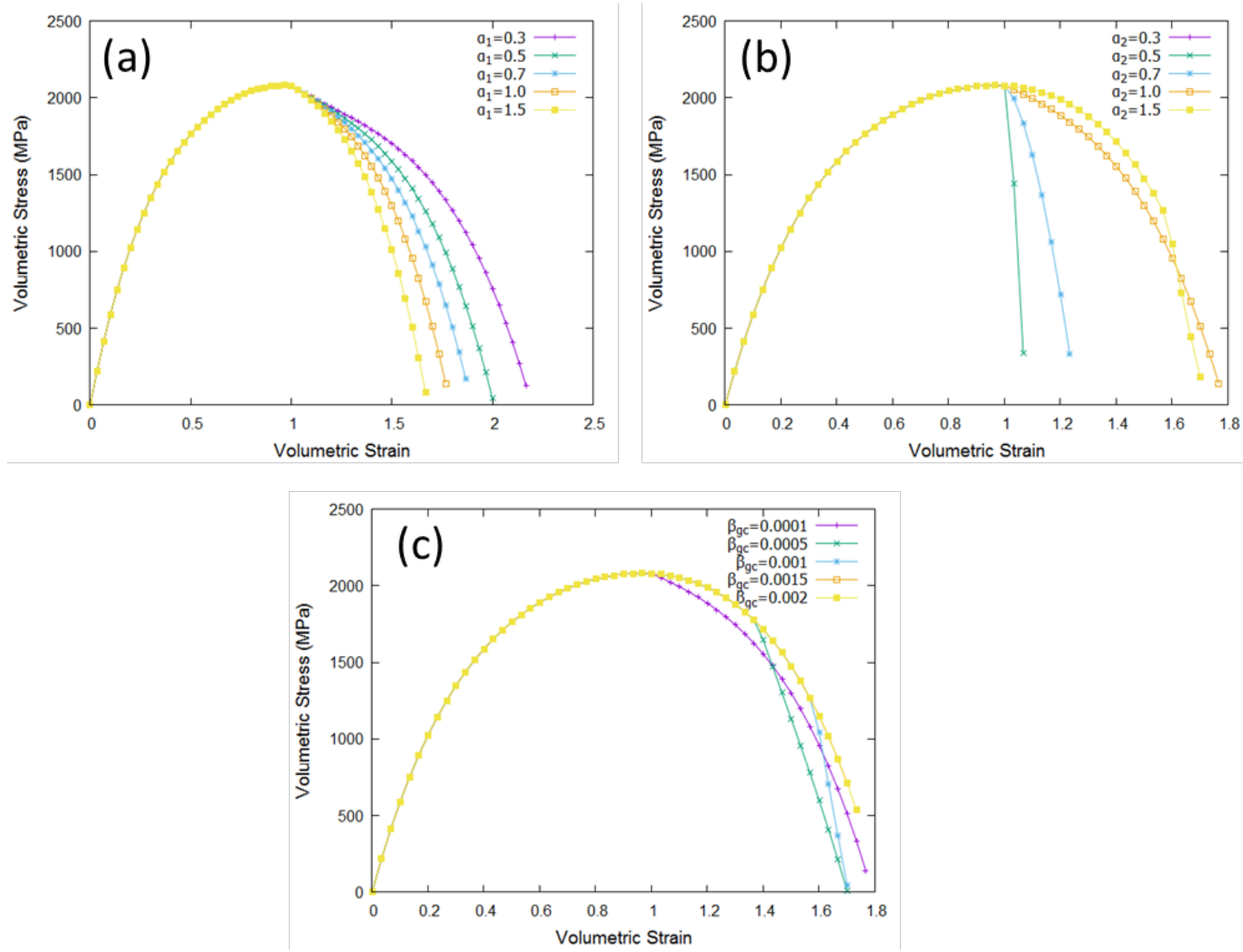

Figure 4: Parametric study for the case of uniaxial loading with void growth and coalescence: Effects of (a) $\alpha_{1}$, (b) $\alpha_{2}$, (c) $\beta_{g c}$

Parametric assessment was performed for different triaxialities. The effect of individual parameters is found to be the same as uniaxial loading and has not been repeated here for brevity. Figure 3 shows the model assessment for the different types of loading triaxialities with the set of parameters discussed in Table 1. It can be inferred from Figure 3 that the proposed model captures the effect of triaxiality, i.e. as the triaxiality is increased the material 
shows softer response. Model is also able to capture the effect of earlier initiation of softening due to void growth with increasing triaxialities and this is due to the increased rate of the void growth with increasing triaxialities (as shown in Figure 3 (b)).

Figure 4 shows the parametric assessment of the model once $\beta_{g}$ reaches to a critical value $\left(\beta_{g c}\right)$, i.e. the void growth will be taken over by void coalescence. There are three material parameters which control the coalescence part of the stress-strain curve $\left(\alpha_{1}, \alpha_{2}, \beta_{g c}\right)$ which in general should be identified from experiments. It is found that variation in $\alpha_{1}$ and $\alpha_{2}$ values affect the damage evolution part of the stress-strain curves. It can be inferred from Figure 4 (a) that as the value of $\alpha_{1}$ is increased the slope of the damage evolution part of the stress-strain curve increases. This indicates that increasing the value of $\alpha_{1}$ makes the void coalescence process more sensitive to strain. Figure 4 (b) shows the effect of $\alpha_{2}$ on the stressstrain curves. It is found that with increasing values of $\alpha_{2}$ the slope of the damage evolution part decreases. It is also found that below certain values of $\alpha_{2}$ the void coalescence become highly sensitive to the strain and material failure becomes almost brittle in a relative sense. As discussed above, $\beta_{g c}$ is the critical value for the onset of the coalescence. Figure 4 (c) shows that increasing the $\beta_{g c}$ increases the strain at which void coalescence begin.

\section{Finite Element based Numerical Simulations and Validation}

To check model capability of capturing actual failure phenomena, the presented material model has been implemented in a user defined material subroutine (VUMAT) and interfaced with ABAQUS finite element software. For validation purposes, material parameters are identified for the uniaxial stress-strain data reported in [38] and shown in Figure 5. The results are for [ $\left[\begin{array}{lll}0 & 0 & 1\end{array}\right]$ oriented copper crystals (Table 3) while the identified set of parameters are given in Table 2. The orientation of single crystal is defined using the same analogy as in [3], [31], [39], i.e. by giving components of two non-parallel vectors in local and global system. Results show a good agreement between experiments and simulations (Figure 5).

Table 2: Material parameters identified for porous copper single crystal through inverse modelling

\begin{tabular}{lllllllll}
\hline$B$ & $\varepsilon_{h 0}$ & $\mathrm{~m}_{2}$ & $s$ & $A$ & $\beta_{g c}$ & $\alpha_{1}$ & $\alpha_{2}$ \\
\hline 0.000013 & 1.0 & 1.0 & 43000. & 1.0 & 0.0000018 & 0.1 & 0.95 \\
\hline
\end{tabular}

Table 3: Crystal orientation relationship $\left(1^{\text {st }}\right.$ and $2^{\text {nd }}$ vectors to determine crystal orientation in global system)

\begin{tabular}{|c|l|l|l|l|l|l|l|}
\hline Orientation & & \multicolumn{3}{|c|}{ Direction in local system } & \multicolumn{3}{c|}{ Global system } \\
\hline \multirow{2}{*}[\begin{array}{llll}{0}&{0}&{1}\end{array}]{} & $1^{\text {st }}$ Vector & 1 & 0 & 0 & 1 & 0 & 0 \\
\cline { 2 - 9 } & $2^{\text {nd }}$ Vector & 0 & 0 & 1 & 0 & 0 & 1 \\
\hline \multirow{2}{*}{$\mathrm{A}$} & $1^{\text {st }}$ Vector & 1 & 0 & 0 & 0.7071 & 0.7071 & 0 \\
\cline { 2 - 8 } & $2^{\text {nd }}$ Vector & 0 & 0 & 1 & -0.7071 & 0.7071 & 0 \\
\hline \multirow{2}{*}{$\mathrm{B}$} & $1^{\text {st }}$ Vector & 1 & 0 & 0 & 0 & 0 & 1 \\
\cline { 2 - 8 } & $2^{\text {nd }}$ Vector & 0 & 0 & 1 & 0 & -1 & 0 \\
\hline
\end{tabular}




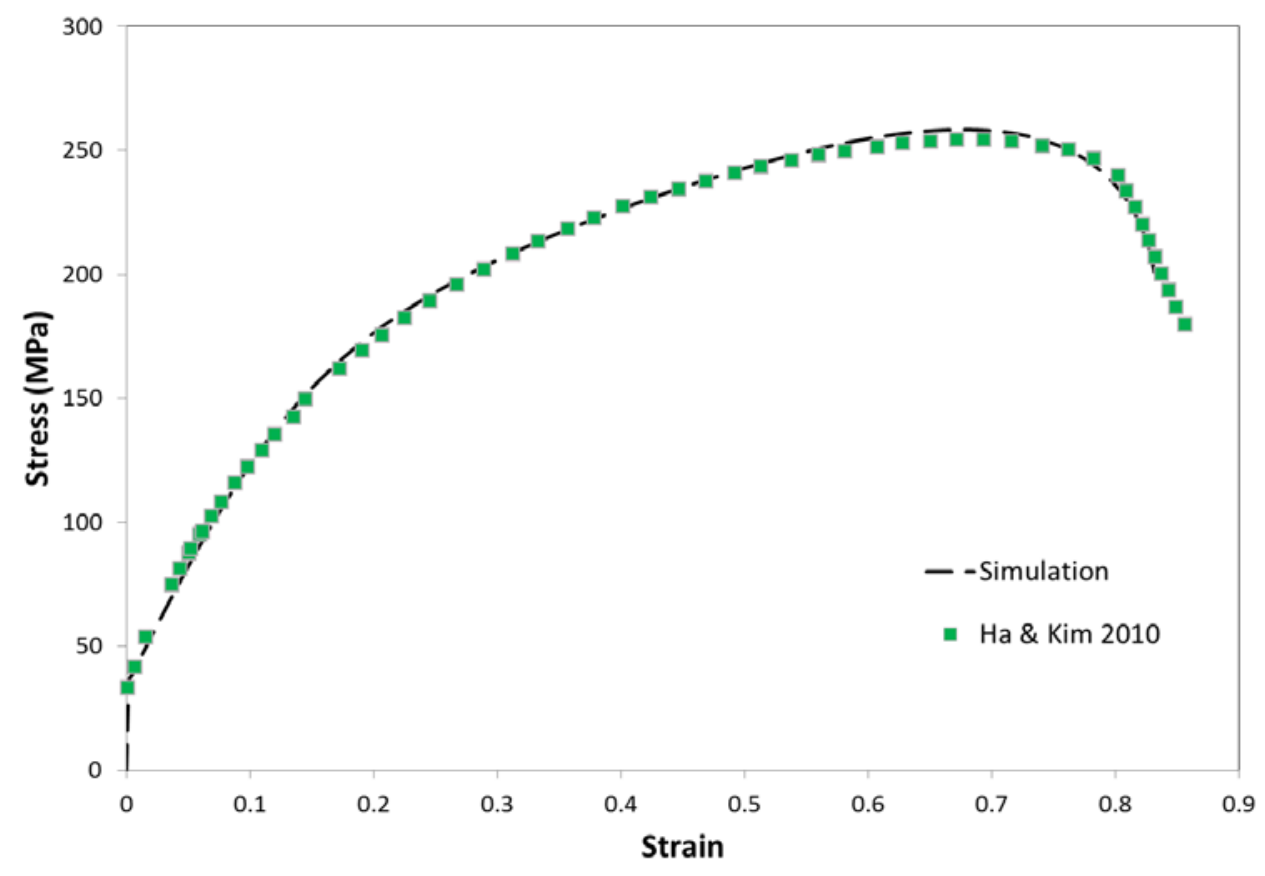

Figure 5: Model predictions after parameter identification of single crystal RVE model with void growth and coalescence for [0 0 1] orientation (Ha and Kim, 2010)

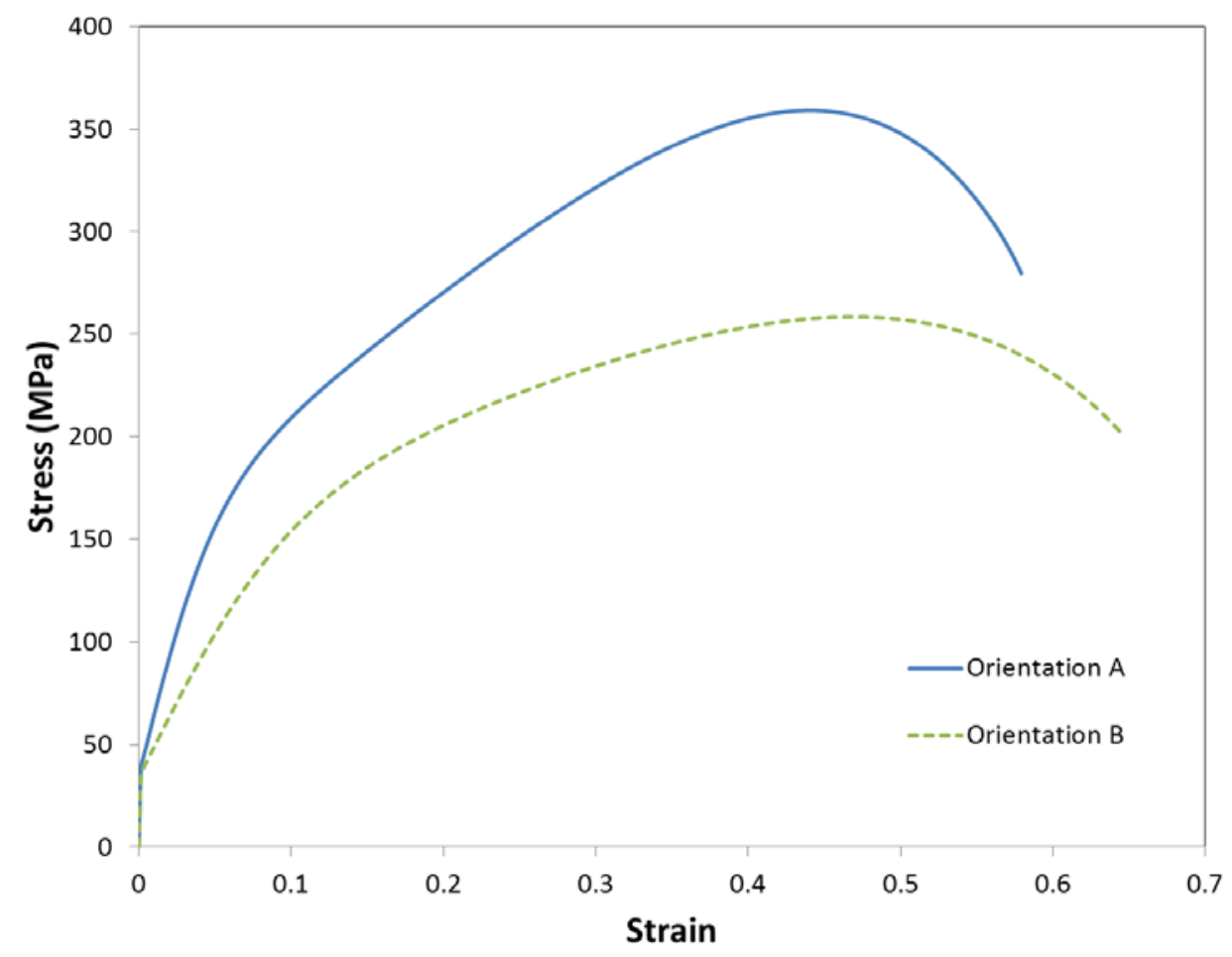

Figure 6: Orientation effect on uniaxial stress-strain curve

It has been reported in the previous representative volume element based studies ([2] and references there in) that initial crystal orientation has a significant effect on void growth and stress-strain curves. In order to demonstrate the model capability of capturing the orientation effect on stress-strain response two different orientations (A and B as shown in Table 3) were selected and model predictions are plotted in Figure 6. It can be inferred from Figure 6 that 
model is clearly able to capture the orientation effect not only in the hardening part but also in the softening part of the stress-strain curves. Model is also able to show the effect of orientation on the final failure (strain to fracture) which has been reported in the literature in the past ([2] and references there in).

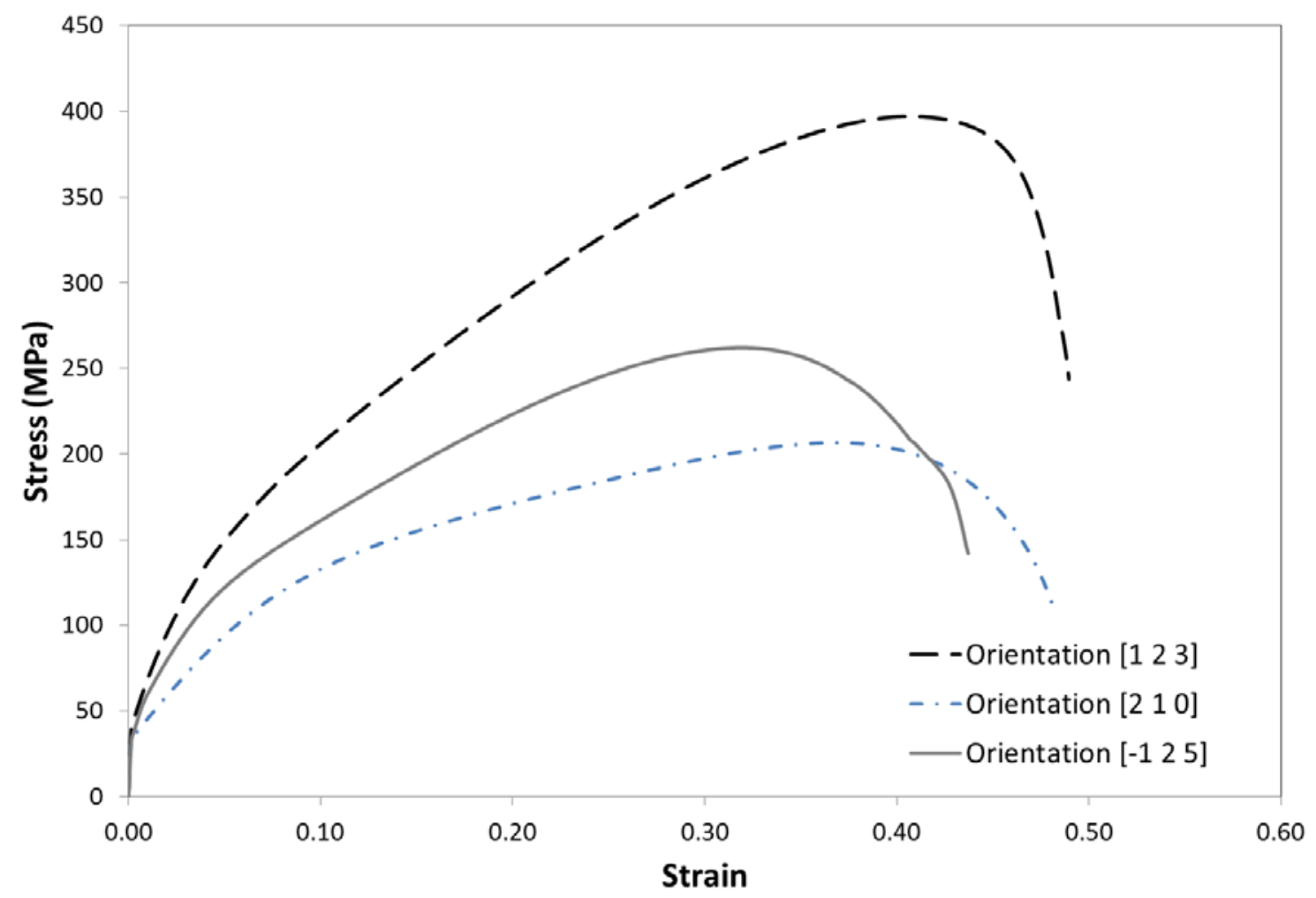

Figure 7: Effect of crystal orientation on the overall stress strain behaviour for three different orientations under uniaxial loading

Further orientations from literature, namely orientation [123] from Ha and Kim [38] and orientations [210] and [125] from Ling et al. [40], are simulated to understand and demonstrate model's response. It is found that peak stress depends on the orientation and qualitatively agrees with unit cell calculations presented in Ha and Kim [38] and Ling et al. [40]. It is found that orientation [123] manifests clearly the highest peak stresses and is also higher than the peak stress for orientation [001] in Figure 5 which was also reported by Ha and Kim [38]. Also, it can be inferred from Figure 7 that softening regime starts earlier for orientation [125] than orientation [210] which is also in conjunction with the results reported by Ling et al. [40].

\section{Conclusions and Future Directions}

A crystal plasticity based constitutive model has been developed which accounts for the damage due to the void growth and coalescence in the single crystalline framework. A detailed parametric assessment has been performed to understand and clarify the effect of individual parameter on material response during deformation. Finite element based applications have been presented by identifying material parameters for available data from literature. It is found that model shows a good agreement with the experiments. The same has 
then been utilised to verify the experimental findings through finite element based modelling of the representative microstructure of the material by predicting fracture due to void growth and coalescence inside the microstructure. As an outlook, there exists a complex relationship among matrix anisotropy (slip, twinning, transformation) and microstructure which is under investigation in the group and will be reported in the future. Further directions for this work will include the application of the proposed constitutive model on sheet metal forming process. This will also include a detailed investigation of the material behaviour for various deformation triaxialities during sheet metal forming processes and look for the ways to develop analytical forming limit curves for a specific material to save time and cost, which is an ongoing work in the research group.

\section{Acknowledgements}

The author thankfully acknowledges the financial support of EPSRC funding (EP/ L021714/1). 


\section{References}

[1] A. Siddiq, R. Arciniega, and T. El Sayed, “A variational void coalescence model for ductile metals,” Comput. Mech., vol. 49, no. 2, pp. 185-195, 2012.

[2] U. Asim, M. A. Siddiq, and M. Demiral, "Void growth in high strength aluminium alloy single crystals: a CPFEM based study,” Model. Simul. Mater. Sci. Eng., vol. 25, no. 3, p. 35010, 2017.

[3] A. Siddiq, S. Schmauder, and Y. Huang, "Fracture of bicrystal metal/ceramic interfaces: A study via the mechanism-based strain gradient crystal plasticity theory,” Int. J. Plast., vol. 23, no. 4, pp. 665-689, 2007.

[4] A. Weck and D. S. Wilkinson, "Experimental investigation of void coalescence in metallic sheets containing laser drilled holes,” Acta Mater., vol. 56, no. 8, pp. 1774-1784, 2008.

[5] Y. Lou, H. Huh, S. Lim, and K. Pack, "New ductile fracture criterion for prediction of fracture forming limit diagrams of sheet metals,” Int. J. Solids Struct., vol. 49, no. 25, pp. 3605-3615, 2012.

[6] D. Fabrègue and T. Pardoen, "A constitutive model for elastoplastic solids containing primary and secondary voids,” J. Mech. Phys. Solids, vol. 56, no. 3, pp. 719-741, 2008.

[7] K. Weinberg, A. Mota, and M. Ortiz, “A variational constitutive model for porous metal plasticity.,” Comput. Mech., vol. 37, pp. 142-152, 2006.

[8] F. A. McClintock, “A Criterion for Ductile Fracture by the Growth of Holes,” ASME - J. Appl. Mech., vol. 35, pp. 363-371, 1968.

[9] J. R. Rice and D. M. Tracey, "On the Ductile Enlargement of Voids in Triaxial Stress Fields.," J. Mech. Phys. Solids, vol. 17, pp. 201-217, 1969.

[10] A. L. Gurson, "Continuum Theory of Ductile Rupture by Void Nucleation and Growth: Part I Yield Criteria and Flow Rules for Porous Ductile Media," J. Eng. Mater. Technol., vol. 99, pp. 2-15, 1977.

[11] J. Koplik and a. Needleman, "Void growth and coalescence in porous plastic solids," Int. J. Solids Struct., vol. 24, no. 8, pp. 835-853, 1988.

[12] V. Tvergaard and A. Needleman, "Analysis of the cup-cone fracture in a round tensile bar.," Acta Metall., vol. 32, pp. 157-169, 1984.

[13] T. Pardoen, I. Doghri, and F. Delannay, "Experimental and numerical comparison of void growth models and void coalescence criteria for prediction of ductile fracture in copper bars.," Acta Metall., vol. 46, pp. 541-552, 1998.

[14] X. Han, J. Besson, S. Forest, B. Tanguy, and S. Bugat, "A yield function for single crystals containing voids,” Int. J. Solids Struct., vol. 50, no. 14-15, pp. 2115-2131, 2013.

[15] T. Schacht, N. Untermann, and E. Steck, "The influence of crystallographic orientation on the deformation behaviour of single crystals containing microvoids,” Int. J. Plast., vol. 19, no. 10, pp. 1605-1626, Oct. 2003.

[16] T. L. O’Regan, D. F. Quinn, M. a. Howe, and P. E. McHugh, "Void growth simulations in single crystals," Comput. Mech., vol. 20, no. 1-2, pp. 115-121, 1997.

[17] a. Srivastava and a. Needleman, "Void growth versus void collapse in a creeping single crystal,” J. Mech. Phys. Solids, vol. 61, no. 5, pp. 1169-1184, 2013. 
[18] S. K. Yerra, C. Tekog̃lu, F. Scheyvaerts, L. Delannay, P. Van Houtte, and T. Pardoen, "Void growth and coalescence in single crystals," Int. J. Solids Struct., vol. 47, no. 7-8, pp. 10161029, 2010.

[19] a Srivastava and a Needleman, "Porosity evolution in a creeping single crystal," Model. Simul. Mater. Sci. Eng., vol. 20, no. 3, p. 35010, 2012.

[20] M. Huang, Z. Li, and C. Wang, "Discrete dislocation dynamics modelling of microvoid growth and its intrinsic mechanism in single crystals," Acta Mater., vol. 55, no. 4, pp. 13871396, 2007.

[21] C. Mi, D. a. Buttry, P. Sharma, and D. a. Kouris, "Atomistic insights into dislocation-based mechanisms of void growth and coalescence,” J. Mech. Phys. Solids, vol. 59, no. 9, pp. 18581871, 2011.

[22] A. M. K. D. A. Constantinescu, "A homogenization based yield criterion for a porous Tresca material with ellipsoidal voids,” Int. J. Fract., vol. 200, pp. 209-225, 2016.

[23] D. Song and P. P. Castañeda, "A finite-strain homogenization model for viscoplastic porous single crystals : I - Theory,” vol. 0, pp. 1-20, 2017.

[24] A. Mbiakop, A. Constantinescu, and K. Danas, "An analytical model for porous single crystals with ellipsoidal voids,” J. Mech. Phys. Solids, vol. 84, pp. 436-467, 2015.

[25] J. Paux, L. Morin, R. Brenner, and D. Kondo, “An approximate yield criterion for porous single crystals,” Eur. J. Mech. / A Solids, vol. 51, pp. 1-10, 2015.

[26] J. Besson, Continuum models of ductile fracture: A review Continuum Models of Ductile. 2011.

[27] A. A. Benzerga and J.-B. Leblond, "Ductile Fracture by Void Growth to Coalescence,” 2010, pp. 169-305.

[28] A. A. Benzerga, J. B. Leblond, A. Needleman, and V. Tvergaard, Ductile failure modeling, vol. 201, no. 1. 2016.

[29] U. Asim, M. A. Siddiq, and M. Demiral, "Void growth in high strength aluminium alloy single crystals: a CPFEM based study,” Model. Simul. Mater. Sci. Eng., vol. 25, no. 3, p. 35010, 2017.

[30] R. Hill and J. R. Rice, “Constitutive analysis of elastic-plastic crystals at arbitrary strain,” $J$. Mech. Phys. Solids, vol. 20, no. 6, pp. 401-413, Dec. 1972.

[31] Y. Huang, "A User-Material Subroutine Incorporating Single Crystal Plasticity in the ABAQUS Finite Element Program,” Mech Rep. 178, no. June, pp. 1-47, 1991.

[32] A. Siddiq and S. Schmauder, "Interface fracture analyses of a bicrystal niobium/alumina specimen using a cohesive modelling approach,” Model. Simul. Mater. Sci. Eng., vol. 14, no. 6, p. 1015, 2006.

[33] D. Peirce, R. J. Asaro, and a. Needleman, "An analysis of nonuniform and localized deformation in ductile single crystals,” Acta Metall., vol. 30, no. 6, pp. 1087-1119, 1982.

[34] A. A. Benzerga and J. B. Leblond, "Effective Yield Criterion Accounting for Microvoid Coalescence,” J. Appl. Mech. Asme, vol. 81, no. 3, pp. 4-7, 2014.

[35] M. E. Torki, A. A. Benzerga, and J.-B. Leblond, “On Void Coalescence Under Combined Tension and Shear,” J. Appl. Mech., vol. 82, no. 7, p. 71005, 2015. 
[36] A. A. Benzerga, "Micromechanics of Coalescence in Ductile Fracture,” J. Mech. Phys. Solids, vol. 50, pp. 1331-1362, 2002.

[37] F. Scheyvaerts and T. Pardoen, "A new model for void coalescence by internal necking.,” Int. J. Damage Mech., vol. 19, pp. 95-126, 2010.

[38] S. Ha and K. Kim, "Void growth and coalescence in f.c.c. single crystals," Int. J. Mech. Sci., vol. 52, no. 7, pp. 863-873, 2010.

[39] A. Siddiq and T. E. Sayed, "A thermomechanical crystal plasticity constitutive model for ultrasonic consolidation,” Comput. Mater. Sci., vol. 51, no. 1, pp. 241-251, 2012.

[40] C. Ling, J. Besson, B. Tanguy, F. Latourte, and E. Bosso, "An elastoviscoplastic model for porous single crystals at finite strains and its assessment based on unit cell simulations," Int. J. Plast., vol. 84, pp. 58-87, Sep. 2016. 